\title{
OPTIMAL POWER ALLOCATION IN OPPORTUNISTIC RELAYING WITH OUTDATED CSI
}

\author{
Jose Lopez Vicario, Antoni Morell, Albert Bel, Gonzalo Seco-Granados \\ Group of Signal Processing for Communications and Navigation (SPCOMNAV) \\ Universitat Autonoma de Barcelona (UAB) \\ Edifici Q - Campus UAB, 08193 Bellaterra (Barcelona), Spain \\ Phone: +34 9358135 62, Fax: +34 935814031 \\ e-mail:\{jose.vicario, antoni.morell, albert.bel, gonzalo.seco\}@uab.es
}

\begin{abstract}
In this paper, we study the impact of outdated channel state information (CSI) on a cooperative system based on opportunistic relay selection (ORS). The study is carried out by obtaining an analytical expression for the outage probability, defined as the probability that the instantaneous mutual information is lower than a target rate. Besides, we propose the optimal power allocation aimed at minimizing the outage probability when the available CSI is subject to impairments. As shown in the paper, the proposed strategy provides significant gains when compared with uniform power allocation.
\end{abstract}

\section{INTRODUCTION}

Cooperative diversity has been shown to be an efficient way to combat wireless impairments using low complexity terminals $[1,2,3]$. Basically, these schemes allow for the exploitation of spatial diversity gains without the need of multi-antenna technology. Different spatial paths are provided by sending/receiving the information to/from a set of cooperating terminals working as relays. By doing so, most of the advantages of multiple-input multiple-output (MIMO) techniques [4] can be extracted while keeping the complexity of the individual terminals reduced.

Among the set of cooperative techniques, opportunistic relay selection (ORS) is a useful strategy for practical implementation [5]. This is because ORS is a low complexity strategy consisting in only activating the best relay (in accordance with performance metric). Apart from the inherent simplicity of the proposed technique, this strategy avoids the need of synchronization (needed by most distributed space-time coding schemes) and reduces the power consumption of the terminals.

When ORS is implemented in a real system, however, there may exist a delay between the instants when the selection process is encompassed and the actual transmission of data from the selected relay takes place. In other words, the channel state of the selected relay considered at the selection decision can substantially differ from the actual one and, as a result, system performance is affected.

The study of the impact of outdated channel state information (CSI) on ORS has been addressed by very few authors. For instance, it was shown in [6] that a selection relaying mechanism based on localization knowledge can outperform an opportunistic scheme with instantaneous information. Although it was not explicitly discussed, the reason for that being that available CSI was subject to delays. As a consequence, the selection scheme proposed in [6] may work better when decisions are made based on location information instead of instantaneous but outdated CSI (localization variations are considerably slower than those induced by the wireless channel).

In this paper, we analytically study the effect of outdated CSI on the outage probability of a ORS system based on decode and forward. Besides, we derive the power allocation strategy aimed at minimizing the outage probability as a function of the level of CSI degradation. As shown in the paper, the proposed strategy significantly improves the system performance, specially when the CSI uncertainty is outdated.

\section{SYSTEM MODEL}

Consider a wireless network where one mobile unit (source) sends information to the base station (destination). In order to improve system performance, a cooperative mechanism is considered. In particular, an ORS strategy is adopted in a scenario with $K$ mobile units of the network working as relays.

For the sake of notation simplicity, we define an arbitrary link $A$ - $B$ between two nodes $A$ and $B$. Node $A$ can be the source $(A=S)$ or the $k$-th relay $(A=k)$, whereas node $B$ can correspond to the $k$-th relay ( $B=k$ ) or to the destination 
$(B=D)$. With this model in mind, the received signal in the link $A-B$ can be written as follows:

$$
r_{B}=h_{A, B} x_{A}+n_{B}
$$

where $x_{A} \in \mathbb{C}$ is the transmitted symbol from node $A$ with power $P_{A}=\mathbb{E}\left[\left|x_{A}\right|^{2}\right], n_{B} \in \mathbb{C}$ is AWGN noise with zero mean and variance $\sigma_{n}^{2}$ (independent of the value of $B), h_{A, B} \in \mathbb{C}$ is the channel response between nodes $A$ and $B$ modeled as $h_{A, B} \sim C N(0,1)$ (Rayleigh fading). We assume a block-fading channel where the channel response remains constant during one time-slot and that the different channels (for changing A or B) are independent and identically distributed (i.i.d.). Concerning power allocation, we consider that total transmit power of the system, $P$, remains constant and is distributed among the source and the selected relay, $k^{*}$, as $P_{S}=(1-\alpha) P$ and $P_{k^{*}}=\alpha P$. We denote by $\gamma_{A, B}=P_{A}\left|h_{A, B}\right|^{2} / \sigma_{n}^{2}$ the instantaneous signalto-noise ratio (SNR) experienced in the link $A$ - $B$ in a given time-slot and we define $\bar{\gamma}=P / \sigma_{n}^{2}$ as the system SNR.

Concerning the relaying procedure, we consider a halfduplex two-hop decode and forward (DF) protocol as relaying strategy. When using half-duplex DF, the transmission is divided in two time-slots. In the first time-slot, the source transmits the information to the relays, which attempt to demodulate and decode this information. In the second timeslot, the relays encode again the information and retransmit it to the destination [3]. In an ORS scheme, only the best relay is allowed to cooperate with the source. More specifically, the subset of relays able to decode the information is named as the decoding subset $\mathcal{D S}$ and, from that subset, the relay with the best relay-to-destination channel quality retransmits the information (see Fig. 1). In particular, the relay maximizing the SNR in the link $k$ - $D$ is selected:

$$
k^{*}=\arg \max _{k \in \mathcal{D} \mathcal{S}}\left\{\hat{\gamma}_{k, D}\right\}
$$

where $\hat{\gamma}_{k, D}$ is the SNR available at the relay selection instant, which can differ from the actual SNR $\gamma_{k, D}$ during information transmission due to channel variations. Indeed, we assume that $\hat{\gamma}_{k, D}$ were obtained from channel state $\hat{h}_{k, D}$ (i.e., $\hat{\gamma}_{k, D}=\alpha P\left|\hat{h}_{k, D}\right|^{2} / \sigma_{n}^{2}$ ), which is an outdated version of $h_{k, D}$. Then, these two random variables are jointly Gaussian and one can easily show that $h_{k, D}$ conditioned on $\hat{h}_{k, D}$ follows a Gaussian distribution [7]:

$$
h_{k, D} \mid \hat{h}_{k, D} \sim \mathcal{C N}\left(\rho \hat{h}_{k, D}, 1-\rho^{2}\right)
$$

where parameter $\rho$ is the correlation coefficient between $\hat{h}_{k, D}$ and $h_{k, D}$, having different values according with the channel model ${ }^{1}$. From the above discussion, it is straightforward to show that the actual SNR, $\gamma_{k, D}$, conditioned on

\footnotetext{
${ }^{1}$ For the sake of mathematical tractability, we assume a scenario where parameter $\rho$ is the same for all the relays.
}

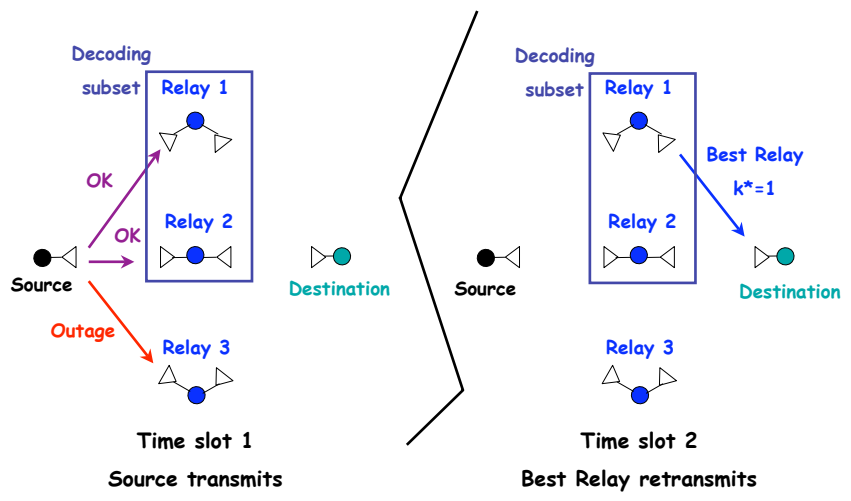

Fig. 1. Cooperative communications scheme based on ORS with DF.

its estimate, $\hat{\gamma}_{k, D}$, follows a non-central chi-square distribution with 2 degrees of freedom, whose probability density function (pdf) takes the following expression [7]:

$$
\begin{aligned}
& f_{\gamma_{k, D} \mid \hat{\gamma}_{k, D}}\left(\gamma_{k, D} \mid \hat{\gamma}_{k, D}\right)=\frac{1}{\alpha \bar{\gamma}\left(1-\rho^{2}\right)} e^{\frac{-\left(\gamma_{k, D}+\rho^{2} \hat{\gamma}_{k, D}\right)}{\alpha \bar{\gamma}\left(1-\rho^{2}\right)}} \\
& \times I_{0}\left(\frac{2 \sqrt{\rho^{2} \gamma_{k, D} \hat{\gamma}_{k, D}}}{\alpha \bar{\gamma}\left(1-\rho^{2}\right)}\right)
\end{aligned}
$$

with $I_{0}(\cdot)$ standing for the zero-order modified Bessel function of the first kind.

\section{OUTAGE PROBABILITY}

We define the outage probability as the probability that the instantaneous mutual information of the system is below a predefined value $R$. Since we consider a two-hop DF scenario, we should start the analysis by studying the decoding subset $\mathcal{D S}$, i.e. the subset of relays that are not in outage when considering the source-to-relay link:

$\mathcal{D S}=\left\{k: \log _{2}\left(1+\gamma_{S, k}\right) \geq 2 R\right\}=\left\{k: \gamma_{S, k} \geq 2^{2 R}-1\right\}$

Note that we have considered that outage in the first hop occurs when instantaneous capacity is lower than $2 R$ (as it will be done in the relay-to-destination link). By doing so, the resulting end-to-end spectral efficiency is $R$ as the proposed two-hop scheme requires two time-slots to transmit the information from the source to the destination.

In order to obtain the outage probability of the system, the probability that the selected relay is in outage must be computed for each combination of relays in the decoding subset. By defining now $\mathcal{D} \mathcal{S}_{l}$ as an arbitrary decoding subset with $l$ relays, we can easily compute the outage proba- 
bility as [5]:

$$
P_{\text {out }}(y, \alpha, \rho)=\sum_{l=0}^{K} \sum_{\mathcal{D} \mathcal{S}_{l}} \operatorname{Prob}\left(\text { outage } \mid \mathcal{D S} \mathcal{S}_{l}\right) \operatorname{Prob}\left(\mathcal{D S}_{l}\right)
$$

where the second summation is over all the possible decoding subsets $\mathcal{D} \mathcal{S}_{l}$ (i.e., the $\left(\begin{array}{c}K \\ l\end{array}\right)$ possible subsets of $l$ relays taken from the $K$ relays) and $\operatorname{Prob}\left(\right.$ outage $\mid \mathcal{D S} \mathcal{S}_{l}$ ) is the probability of outage in the relay-to-destination link conditioned on that the decoding subset is $\mathcal{D} \mathcal{S}_{l}$, being $\operatorname{Prob}\left(\mathcal{D} \mathcal{S}_{l}\right)$ the probability of that subset. According to the proposed homogeneous scenario (all channels are i.i.d) with outdated CSI, the outage probability can be written as follows (further details are provided in the Appendix):

$$
\begin{aligned}
& P_{\text {out }}(y, \alpha, \rho)=\left(1-e^{-\frac{y}{(1-\alpha) \gamma}}\right)^{K} \\
& \quad+\sum_{l=1}^{K} l \sum_{m=0}^{l-1}\left(\begin{array}{c}
l-1 \\
m
\end{array}\right) \frac{(-1)^{m}}{m+1}\left(1-e^{-y \frac{m+1}{\alpha \bar{\gamma}\left(1+m\left(1-\rho^{2}\right)\right)}}\right) \\
& \left(\begin{array}{c}
K \\
l
\end{array}\right)\left(1-e^{-\frac{y}{(1-\alpha) \bar{\gamma}}}\right)^{K-l} e^{-\frac{y l}{(1-\alpha) \bar{\gamma}}}
\end{aligned}
$$

where one should recall the Rayleigh fading assumption, note that the first term is related with the case that the decoding subset is an empty subset (i.e., $l=0$ ) and that $y$ has been defined as $y=2^{2 R}-1$ for the sake of notation simplicity.

\section{OPTIMAL POWER ALLOCATION}

In this paper, we consider the optimal power allocation as the power allocation minimizing the outage probability, that is:

$$
\begin{array}{cc}
\min _{\alpha} & P_{\text {out }}(y, \alpha, \rho) \\
\text { s.t. } & 0<\alpha<1
\end{array}
$$

The optimal solution to the above minimization problem can be efficiently obtained with the help of classical nonlinear optimization techniques, such as the gradient method or the section methods [8, Chapter 2], as it is a quasi-convex problem (further details are provided in the Appendix). In this work, in particular, we have considered the golden section method to solve the power allocation problem.

In order to give some insight on the physical nature of the problem, we present in Fig. 3 the outage probability as a function of power parameter $\alpha$ for different values of $\rho$ in a scenario with $K=5$ relays and target rate $R=1 \mathrm{bit} / \mathrm{s} / \mathrm{Hz}$. For each of them, we also indicate the optimum value of $\alpha$ minimizing outage capacity. The behavior of the optimal power allocation is that the optimal value of $\alpha$ grows for decreasing values of $\rho$. In other words, more power is assigned to the selected relay when the estimate of the SNR becomes less accurate. The physical interpretation of such an effect is the following:

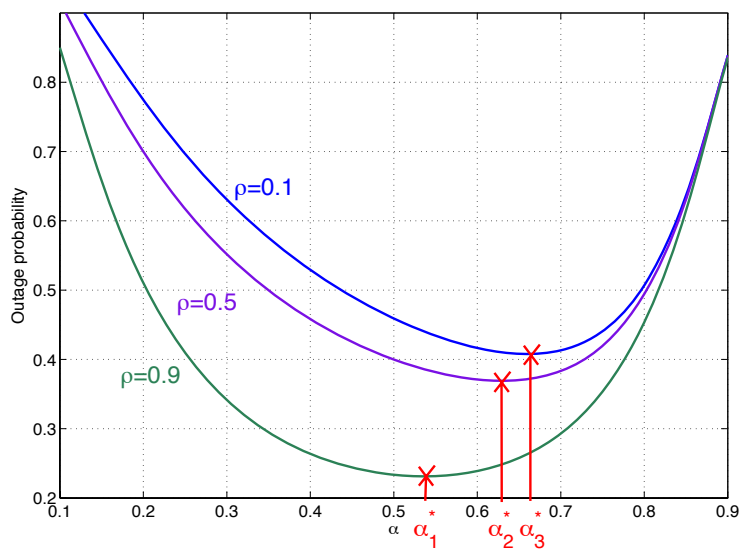

Fig. 2. Outage probability vs. $\alpha$ for different values of $\rho$ ( $K=5$ relays, $R=1 \mathrm{bit} / \mathrm{s} / \mathrm{Hz}, \bar{\gamma}=10 \mathrm{~dB}$ ).

- For decreasing values of $\rho$, the accuracy of the relay selection algorithm is affected. Then, the quality of the $k^{*}-D$ link can be far from being satisfactory. In that case, it is better to give more power to the selected relay in order to improve the reliability of that link. Allocating more power to the source would increase the number of nodes belonging to the decoding subset, being this measure not practical as relay selection tends to be random.

- For increasing values of $\rho$, selection algorithm is more accurate and then it is interesting to have a good balance between the source and relay powers. On the one hand, having a higher number of relays in the decoding subset increases the chances of having a very good relay in $\mathcal{D S}$. On the other hand, assigning more power to the selected relay is beneficial for exploiting the fact that a good link is selected. Indeed for $\rho=1$ the optimal value of $\alpha$ is $\alpha^{*}=0.5$, i.e. the optimal choice is uniform power allocation.

\section{NUMERICAL EVALUATION}

As far as the numerical evaluation is concerned, we have advanced some results in Fig. 3. Apart from the behavior of the optimal power allocation, one can also observe in that figure the gain achieved with the proposed strategy with respect to uniform power allocation $(\alpha=0.5)$. When $\rho=0.1$, for instance, the proposed strategy reduces the outage probability approximately $11 \%$.

In order to facilitate the comparison, we also present outage probability results obtained for different values of $\rho(\rho=0.1,0.5,0.8$ and 1$)$ and for different power allocation strategies as a function of the system SNR. First of all, one can observe that the theoretical expression given by (2) 


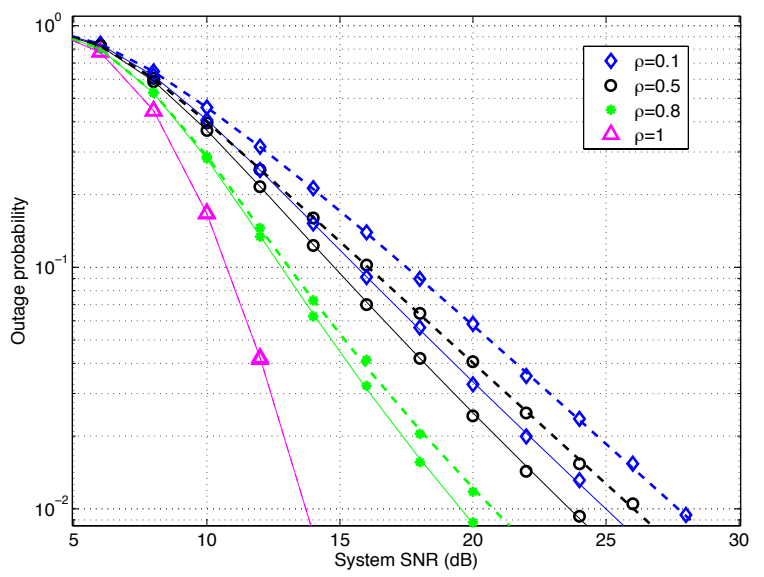

Fig. 3. Outage probability vs. System SNR $\bar{\gamma}$ for different values of $\rho$ and different power allocation strategies $(K=5$ relays, $R=1 \mathrm{bit} / \mathrm{s} / \mathrm{HZ}$, Curve: Theoretical, Symbol: Simulation, Solid: Optimal Power Allocation, Dashed: Uniform Power Allocation ).

completely agrees with the simulated curves. Besides, it is shown the gain obtained with the optimal power allocation strategy. For $\rho=1$, the optimal allocation strategy tends to the uniform one and, as a result, the same results are observed for both strategies. However, as soon as the accuracy of the CSI information is affected, higher is the benefit observed with the proposed power allocation strategy. For an outage probability target equal to $10 \%$, for instance, 1.2 and $2 \mathrm{~dB}$ gains are obtained with the optimal power allocation strategy when $\rho$ is equal to 0.5 and 0.1 , respectively. Indeed, the optimal strategy gain is emphasized when outage probability requirement are more restrictive. When this value is set to $1 \%$, the gain is also significant for high $\rho$ values. As shown in the figure, the gain attained with the proposed strategy amounts to $2 \mathrm{~dB}$ when $\rho=0.5$ and $3 \mathrm{dBs}$ in the case that $\rho$ is equal to 0.1 .

\section{CONCLUSIONS}

In this work, we have analyzed the impact of outdated CSI on ORS. To do so, we have analytically studied the outage probability of the proposed system based on decode and forward. Besides, we have derived the optimal power allocation strategy. As we have shown in the paper, the optimal strategy consists in giving more power to the selected relay when the available CSI is less accurate. By doing so, superior performance can be obtained, specially when outage probability requirements are more restrictive. Conversely, when the CSI becomes more precise the power strategy tends to the uniform power allocation.

\section{APPENDIX}

In this appendix we provide the analytical derivation of the outage probability expression given by (2) and the proof of the quasi-convexity of the power allocation problem.

\subsection{Outage probability expression}

We start the proof by noting that both the values of $\operatorname{Prob}(\mathcal{D S})$ and $\operatorname{Prob}($ outage $\mid \mathcal{D S}$ ) in (1) are the same for all the decoding subsets $\mathcal{D S} \in \mathcal{D S} \mathcal{S}_{l}$. In other words, these probabilities are independent of the combination of relays forming $\mathcal{D S}$ (for a given $l$ ). The reason for that being that we assume an homogenous scenario where all the channels are i.i.d. As a consequence, these probabilities only depend on the number of active relays (i.e., the cardinality of $\mathcal{D S}, \operatorname{card}(\mathcal{D S})$ ) and we can re-writte (1) as:

$P_{\text {out }}(y)=\sum_{l=0}^{K} \operatorname{Prob}($ outage $\mid \operatorname{card}(\mathcal{D S})=l) \operatorname{Prob}(\operatorname{card}(\mathcal{D S})=l)$

where $\operatorname{Prob}(\operatorname{card}(\mathcal{D S})=l)$ is the probability that the decoding subset has $l$ relays, i.e. $K-l$ relays are in outage when the source-to-relay link is considered. By recalling the Rayleigh fading assumption, one can obtain this probability as follows:

$\operatorname{Prob}(\operatorname{card}(\mathcal{D S})=l)=\left(\begin{array}{c}K \\ l\end{array}\right)\left(1-e^{-\frac{y}{(1-\alpha) \bar{\gamma}}}\right)^{K-l} e^{-\frac{y l}{(1-\alpha) \bar{\gamma}}}$

Notice that the above expression does not depend on the correlation parameter $\rho$. This is because a node belongs to the decoding set if it has perfectly decoded the information, which is independent of relay selection decisions.

Concerning $\operatorname{Prob}($ outage $\mid \operatorname{card}(\mathcal{D S})=l)$, this is the probability that the selected relay (out of $l$ relays in $\mathcal{D S}$ ) is in outage. Clearly, this probability is equal to one when there are no relays to retransmit the information (i.e., $\operatorname{card}(\mathcal{D S})=0$ ). For $l>0, \operatorname{Prob}($ outage $\mid \operatorname{card}(\mathcal{D S})=l$ ) can be obtained by noticing that this is a similar problem to that observed in a multi-user scenario where the user with the highest SNR is selected for transmission but available CSI is subject to delays [7]. With this in mind, we can use the cumulative density function (CDF) of the scheduled user's SNR obtained in [7, Eq. 8] to compute the probability that the selected relay is in outage (i.e., its SNR is lower than $y=2^{2 R}-1$ ):

$$
\begin{aligned}
& \operatorname{Prob}(\text { outage } \mid \operatorname{card}(\mathcal{D S})=l)= \\
& l \sum_{m=0}^{l-1}\left(\begin{array}{c}
l-1 \\
m
\end{array}\right) \frac{(-1)^{m}}{m+1}\left(1-e^{-y \frac{m+1}{\alpha \bar{\gamma}\left(1+m\left(1-\rho^{2}\right)\right)}}\right) \quad l>0
\end{aligned}
$$

Finally, by plugging (5) and (4) into (3) and noting that the first term is related to the case that $l=0$, one can easily verify that expression (2) holds. 


\subsection{Quasi-convexity of the power allocation problem}

The power allocation problem is quasi-convex if the outage probability in (2) is a unimodal (or quasi-convex) function on $\alpha$, given that the constraint $0<\alpha<1$ defines a convex subset. Therefore, we need to proof the unimodality of $P_{\text {out }}(\alpha)$. Due to space limitations, we just provide an outline of the proof in this paper but further details can be found in [9].

First, let us rewrite (2) as

$P_{\text {out }}(\alpha)=\operatorname{Prob}($ outage $\mid \operatorname{card}(\mathcal{D S})=0) \operatorname{Prob}(\operatorname{card}(\mathcal{D S})=0)$

$+\operatorname{Prob}(\operatorname{card}(\mathcal{D S})>0) \operatorname{Prob}($ outage $\mid \operatorname{card}(\mathcal{D S})>0)$

$=(1-f(\alpha))+g(\alpha) \cdot f(\alpha)$

where we have used the equality $\operatorname{Prob}($ outage $\mid \operatorname{card}(\mathcal{D S})=$ $0)=1$ and the functions $f(\alpha)$ and $g(\alpha)$ have been defined as:

$$
\begin{aligned}
& f(\alpha)=\operatorname{Prob}(\operatorname{card}(\mathcal{D S})>0) \\
& g(\alpha)=\operatorname{Prob}(\text { outage } \mid \operatorname{card}(\mathcal{D S})>0)
\end{aligned}
$$

respectively. Note that within that representation, $f(\alpha)$ and $g(\alpha)$ are decreasing functions on $\alpha$. In particular,

$$
f(\alpha)=1-\left(1-e^{-\frac{y}{(1-\alpha) \bar{\gamma}}}\right)^{K}
$$

and $g(\alpha)$ is a combination of the outage probabilities given a fixed number of relays in the decoding subset, which is upper-bounded by $\operatorname{Prob}($ outage $\mid \operatorname{card}(\mathcal{D S})=1)=1-$ $e^{-\frac{y}{\gamma \alpha}}$.

Since $P_{\text {out }}(\alpha)$ is bounded between 0 and 1 with $P_{\text {out }}(0)=$ $P_{\text {out }}(1)=1$, at least one minimum is guaranteed for $0<$ $\alpha<1$. The goal is to proof that there is a single minimum or, in other words, that

$$
P_{\text {out }}^{\prime}(\alpha)=f^{\prime}(\alpha) \cdot[g(\alpha)-1]+g^{\prime}(\alpha) \cdot f(\alpha)=0
$$

is attained at a single $\alpha$ value. To do so, the condition in (7) can be alternatively written as

$$
\frac{f^{\prime}(\alpha)}{f(\alpha)}=\frac{g^{\prime}(\alpha)}{1-g(\alpha)}
$$

Then, it is possible to proof that $\frac{f^{\prime}(\alpha)}{f(\alpha)}$ is a monotone decreasing function on $\alpha$ and also that $\frac{g^{\prime}(\alpha)}{1-g(\alpha)}$ increases monotonically; thus (8) can not be satisfied at more than one $\alpha$ value within the search interval. Equivalently, it can be shown that $f(\alpha)$ and $1-g(\alpha)$ are log-concave functions [ $[10$,
Chapter 3] and therefore the function $\log f(\alpha)+\log (1-g(\alpha))$ has a single maximum, which is the optimal power allocation.

\section{REFERENCES}

[1] A.Sendonaris, E. Erkip, and B. Aazhang, "User cooperation diversity, Part I and II," IEEE Trans. On Comm., vol. 51, no. 11, Nov. 2003.

[2] J. N. Laneman, D. Tse, and G. W. Wornell, “Cooperative diversity in wireless networks: Efficient protocols and outage behaviour," IEEE Trans. On Information Theory, vol. 50, no. 12, pp. 3062-3080, Dec. 2004.

[3] R. U. Nabar, H. Bolcskei, and F. W. Kneubler, "Fading relay channels: Performance limits and space-time signal design," IEEE Journal on Sel. Areas in Comm., vol. 22, no. 6, pp. 1099-1109, Aug. 2004.

[4] D. Gesbert, M. Shafi, D. Shiu, and P. Smith, "From theory to practice: An overview of space-time coded MIMO wireless systems," IEEE Journal of Selected Areas in Comm., vol. 21, no. 3, pp. 281-302, April 2003.

[5] A. Bletsas, H. Shin, and M. Z. Win, "Cooperative communications with outage-optimal opportunistic relaying," IEEE Trans. on Wireless Comm., vol. 6, no. 9, pp. 3450-3460, Sept. 2007.

[6] B. Zhao and M. C. Valent, "Practical relay networks: A generalization of hybrid-ARQ," IEEE Journ. on Sel. Areas in Comm., vol. 23, no. 1, pp. 7-18, Jan. 2005.

[7] J.L. Vicario and C. Antn-Haro, "Analytical assessment of multi-user vs. spatial diversity trade-offs with delayed channel state information," IEEE Communications Letters, vol. 10, no. 8, pp. 588-590, Aug. 2006.

[8] W. Sun and Y. Yuan, Optimization Theory and Methods: Nonlinear Programming, Springer, 2006.

[9] J.L. Vicario, A. Morell, A. Bel, and G. Seco-Granados, "Power allocation strategies in opportunistic relaying with outdated CSI," in preparation.

[10] S. Boyd and L. Vandenberghe, Convex optimization, Cambridge University Press, 2004. 